\title{
¿Ciencias duras y ciencias blandas? Una falsa dicotomía ${ }^{1}$
}

\author{
Manuel Gil Antón*
}

Jo comparto, por las razones que expondré más adelante a su consideración obtener ciudadanía científica entre las ciencias de la naturaleza y las ciencias sociales - también llamadas ciencias del hombre. Ni sus formas adjetivadas: duras/ blandas o ciencias "a secas" y "ciencias" sociales.

Considero insostenibles estas dicotomías en nuestros días, y me parece oportuno recordar el esfuerzo weberiano al respecto - realizado hace casi un siglo-, añadiendo a sus indudables logros el aporte del constructivismo genético para reorganizar sus conclusiones e intentar superar —ir más allá - de los linderos tan reiterados de las dicotomías. Sin las demarcaciones mal fundadas, el horizonte de la reflexión epistemológica es más ancho y compartible entre las diversas ciencias, haciendo a un lado fronteras artificiales, murallas innecesarias que impiden la comunicación.

\section{II}

Comparto con varios conocedores de la obra de Jean Piaget que el proyecto central de su obra consistió en la construcción de una epistemología científica. En el Tratado de lógica y conocimiento científico ${ }^{2}$, Piaget señala que la epistemolo-

\footnotetext{
${ }^{1}$ Versión escrita de la Conferencia presentada el 31 de octubre de 2003 en el marco del VIII Foro de Investigación: Congreso Internacional de Contaduría, Administración e Informática, organizado por la División de Investigación de la Facultad de Contaduría y Administración de la UNAM.

*Profesor del Departamento de Sociología de la Universidad Autónoma Metropolitana, Unidad Azcapotzalco. Correo electrónico: maga@correo.azc.uam.mx

2 Jean Piaget, Tratado de lógica y conocimiento científico, volúmenes I y VII, Paidós, Buenos Aires, 1979.
} 
gía contemporánea procura esclarecer los dilemas de la actividad científica ya no desde un sitio ajeno - superior y distante - desde el cual juzgar o normar a las actividades científicas (como ocurrió durante siglos), sino asumiendo las condiciones generales de cientificidad que se exigen a todas ellas: la máxima coherencia lógica en la estructura de la teoría y, si se trata de una ciencia empírica, de una ciencia de realidad como gustaba decirWeber, la aceptación de los protocolos más estrictos en su relación con los fenómenos a los que alude.

Uno de sus más grandes logros, entonces, consistió en la generación y articulación de dos espacios para la validación de su teoría constructivista: el terreno de la sicogénesis de los conocimientos y el tratamiento de la historia de la ciencia como laboratorio epistemológico.

\section{III}

El constructivismo piagetiano distingue, analíticamente, undominio epistemológico interno en cada una de las ciencias, generado por la reflexión en torno a los fundamentos con los que se relacionan los dominios materiales y conceptuales en el proceder científico de que se trate. Son, dice Piaget, los propios especialistas los que enfrentan la tarea crítica de los fundamentos de su actividad particular, y lo hacen tarde o temprano. Si en cada especialidad científica se presenta este esfuerzo, podemos advertir que varios problemas, enfrentados en los diversos campos, resultan comunes a la actividad científica en general y, por ende, propios de un dominio epistemológico general. La epistemología genética, desde este dominio de convergencia en la problemática de las diversas disciplinas, se abre al diálogo con los trabajos de fundamentación de las ciencias y tomando como base su teoría del desarrollo cognitivo - la equilibración (y desequilibración) de las estructuras cognitivas - procura reorganizar — esto es, esclarecer y replantear- los dilemas de la fundamentación de la actividad científica.

\section{IV}

En el terreno de las ciencias sociales, y como parte de la línea de investigación especializada en la epistemología de estas ciencias — que se desarrolla en la Sección de Metodología y Teoría de la Ciencia - llevé a cabo un trabajo de crítica epistemológica a la labor metodológica de uno de los sociólogos más importantes en la historia de esta disciplina: Max Weber. ${ }^{3}$

\footnotetext{
${ }^{3}$ La versión extensa de esta investigación se puede consultar en: Manuel Gil Antón, Conocimiento científico y acción social: crítica epistemológica a la concepción de ciencia en Max Weber, Gedisa, Barcelona, 1997.
} 
Voy a exponer, muy brevemente, el esfuerzo weberiano orientado a la fundamentación del conocimiento científico de la acción social — su trabajo epistemológico interno - con el fin de mostrar la reorganización que es posible proponer desde el constructivismo piagetiano, misma que, a mi juicio, conduce al rechazo de las dicotomías en torno a la construcción de los objetos de conocimiento entre las diversas ciencias.

\section{V}

\section{El argumento weberiano y sus conclusiones: de la acción social como objeto científico a la eterna juventud como destino}

Si atendemos a los Ensayos metodológicos de Max Weber ${ }^{4}$, podemos reconstruir los siguientes pasos:

1. Weber se opone, con mucha fuerza y tenacidad, a la dicotomía radical establecida por la tradición alemana, entre naturaleza y espíritu en materia de actividad científica. Para esa tradición (recordemos a Dilthey) las ciencias de la naturaleza - dado que la naturaleza nos es externa - operan mediante la elaboración de conceptos; por lo contrario, las ciencias histórico-sociales (las del espíritu) —dado que la vida nos es interior - acceden a estos fenómenos a través de la vivencia. Weber se opone a esta visión dicotómica del qué-hacer científico. A su juicio, la vida social es accesible — igual que en toda ciencia - mediante conceptos.

2. Weber también se opone a los que postulan la validez universal del proceder científico derivado del paradigma newtoniano. No está de acuerdo con los que señalan como objetivo de las ciencias histórico-sociales el horizonte restrictivo de las regularidades propias de una "naturaleza humana abstracta".

3. La piedra angular de su crítica, como ha mostrado con toda propiedad entre nosotros Luis F. Aguilar Villanueva ${ }^{5}$, es el rechazo a la idea de la "irracionalidad"

\footnotetext{
${ }^{4}$ Escritos entre 1896 — al arribar a la cátedra de Economía en Heidelberg- y 1920, año de su muerte.

${ }^{5}$ Véase: Luis F. Aguilar Villanueva, Weber: La idea de ciencia social, volumen primero: La tradición y volumen segundo: La innovación, Coordinación de Humanidades, Universidad Nacional Autónoma de México y Miguel Ángel Porrúa, Grupo Editorial, México, 1986. Mi trabajo de estudio sobre la reflexión weberiana es deudor de esta obra, y de los seminarios coordinados por Luis Aguilar en la Maestría de FLACSO sobre este tema en 1984-1986.
} 
—en el sentido de incalculabilidad — de la acción social, muy extendida y dominante en su tradición intelectual. Muestra que la acción social es, al menos, tan calculable —o incalculable (imprevisible) — como la acción de la naturaleza. Insiste que esta supuesta irracionalidad es otra forma de hacer resurgir la dicotomía ontológica entre el "ser" de la naturaleza y el "ser" de la sociedad.

4. Weber está a favor de una idea central: toda actividad científica está mediada - siempre - por la construcción de conceptos. Sin conceptos, no hay actividad científica. Por lo tanto, Weber argumenta que el único criterio válido para distinguir a las ciencias es metodológico: esto es, por la manera en que elaboran sus conceptos. Pero Weber avanza más. Esta diversa manera de elaboración conceptual, depende del interés cognitivo del científico. En otras palabras, la elaboración de los conceptos depende de las preguntas que nos hacemos.

5. En consecuencia, postula la existencia de dos esferas del conocimiento humano, mismas que no dependen de la "naturaleza" de los objetos, puesto que determinar estas supuestas naturalezas es algo imposible después de Kant, sino del interés cognitivo que las anima. Tenemos la esfera de los fenómenos no-interpretables y la esfera de los fenómenos interpretables.

En la primera - la zona donde la interpretación no es requerida por el interés cognitivo - las preguntas se responden mediante el establecimiento de regularidades, en las que el esquema causa-efecto conduce a la explicación, en el sentido de subsumir, sin contradicción, un caso particular en la ley empírica que le corresponde.

En la segunda - la esfera de los fenómenos que requieren interpretación- el interés cognitivo está orientado por preguntas que necesitan ir "más allá" del establecimiento de regularidades. Como parte del mismo proceso científico, además de establecer regularidades, las preguntas empujan al investigador a dar cuenta del sentido que permite comprender la regularidad.

Weber está interesado en lograr, como nos enseña Luis F. Aguilar, una extraordinaria síntesis de dos líneas que corrían paralelas en la tradición alemana: explicar y comprender. Mientras explicar significa, para él, la inclusión del fenómeno particular en una regularidad probabilística basada en el esquema causa-efecto proveniente de la observación, comprender implica la agregación, también conceptual, de la inclusión del fenómeno particular en el esquema medios-fines. Por eso llama al objetivo de la sociología —y de todas las ciencias histórico-sociales - del siguiente modo: explicación comprensiva. 
6. Weber concluye, con mucha coherencia y en contra de buena parte de su tradición intelectual, que en las ciencias histórico-sociales es posible un plus de objetividad: en estas ciencias, podemos ir más allá de la "explicación observadora" sin caer en la metafísica - y arribar a la "explicación interpretativa".

En otras palabras, Weber señala que en las ciencias histórico-sociales hay una sed de explicación causal mayor que en las ciencias de la naturaleza. Y de esto se deriva, como conclusión, una concepción dicotómica - no ontológica, sino metodológica - de la actividad científica. Escuchemos al propio Weber:

Las ciencias de la naturaleza — que no requieren interpretación—operan así:

Podemos observar (en principio, al menos) el comportamiento de las unidades fisiológicas, las células por ejemplo, o cualesquiera elementos psiquicos, tratar de obtener inferencias de esas observaciones, formular reglas ("leyes") para esos comportamientos y "explicar" causalmente con su ayuda procesos particulares, es decir, incluirlos bajo estas leyes ${ }^{6}$

Se observa el comportamiento de las unidades con el fin de intentar la obtención de inferencias, de tal suerte de formular reglas - leyes - para esos comportamientos y, con base en ellas, explicar procesos particulares al incluirlos bajo las regularidades observadas.

Por su parte, las ciencias histórico-sociales están condenadas, en maravillosa frase weberiana, a la eterna juventud, pues:

...siguen una alternancia continua entre:

a.- el intento de ordenar conceptualmente los hechos a través de la formación de conceptos...

b.- la resolución de los cuadros conceptuales así obtenidos mediante la ampliación y el desplazamiento del horizonte de la ciencia y

c.- la formación de nuevos conceptos sobre las bases así transformadas...?

\footnotetext{
${ }^{6}$ Max Weber, Economía y sociedad, Fondo de Cultura Económica, México, 1984, p. 12.

${ }^{7}$ Max Weber, "La 'objetividad' cognoscitiva de la ciencia social y de la política social" (1904), en Ensayos sobre metodologia sociológica, Amorrortu, Buenos Aires, 1993, p. 94.
} 
Y esto genera un ciclo sin fin, siempre abierto. El contraste es muy considerable entre los modos de operar en las dos esferas y sus intereses cognitivos específicos, separados por el requerimiento de la interpretabilidad.

En Economía y sociedad, su obra final, Weber afirma que esto implica que las ciencias histórico-sociales van más allá de la simple determinación de "conexiones y leyes funcionales" propias de las ciencias naturales: este ir más allá les está permanentemente negado a las ciencias de la naturaleza... y es, digámoslo con fuerza, condición de posibilidad para las ciencias de la acción social, particularmente en su caso, la historia y la sociología.

A estas conclusiones arriba Max Weber. Su genialidad y consistencia son sorprendentes y, vistas en su contexto, dignas de gran respeto. Ha pasado casi un siglo - y vaya siglo: ¿son sostenibles ahora? ¿cómo se ubican luego de la revolución científica experimentada en las primeras décadas del siglo XX, y luego del enorme esfuerzo del empirismo lógico y su caída a mitad de la centuria, así como desde la perspectiva del constructivismo genético? A eso dedicaremos el siguiente apartado.

\section{VI}

\section{La reorganización constructivista}

Siguiendo a Piaget, al atender a la historia de la ciencia como laboratorio epistemológico, es menester despejar si la concepción de ciencia natural presente en los trabajos weberianos correspondía, en efecto, a la noción de ciencia natural dominante en su tiempo. Para ello, en la investigación realizada sobre la cual redacto estas cuartillas, fue necesario esclarecer esta cuestión.

Recurrimos al testimonio del físico Ernest Mach, y a los análisis del historiador de la ciencia Pierre Duhem. Con base en ellos, podemos afirmar que, en la época de Weber, la concepción dominante de ciencia natural, especialmente de la física, se caracterizaba por:

- tener como objetivo el establecimiento de regularidades observadas

- dar cuenta de dichas regularidades, esto es, representar al fenómeno y

- describir a las regularidades con el auxilio de las matemáticas. 
Más aún: Mach propone que, para evitar equívocos — dado que era un profundo antimetafísico - la actividad científica debe prescindir de la "equívoca" noción de causa, y sustituirla — pues con ello basta para lo que se propone la ciencia - por la noción de función matemática.

Duhem — curiosamente un pensador que no rechaza a la metafísica - argumenta que, en la actividad científica, lo que se acumula al paso de los años, lo que queda firme y constituye el patrimonio firme de la ciencia, es la descripción de regularidades. A esto lo llama la representación del fenómeno. Sobre este aspecto, firme y claro, los científicos suelen agregar — sin conexión adecuada - explicaciones asociadas a las regularidades. Estas últimas, las explicaciones, suelen desplomarse continuamente pues no provienen del mismo proceder científico. El propio Duhem aporta un ejemplo de esta yuxtaposición arbitraria de regularidades y "explicaciones causales": es el caso de la ley de la refracción simple de la luz, descubierta por Descartes, en la que la regularidad de la constante entre el seno del ángulo de incidencia y el seno del ángulo de refracción es muy valiosa y pervive hasta nuestros días, pero la "explicación" cartesiana es falsa, dado que supone que la luz se transmite instantáneamente a cualquier distancia.

Duhem comenta: a través de la experimentación, la inducción y la generalización se obtiene una ley correcta. Como no se sigue el mismo método para la parte explicativa, se produce el error. En efecto, cuando el astrónomo Danés Römer demostró que la luz se propaga en el espacio a una velocidad finita y medible, se desploma la parte explicativa propuesta por Descartes, pero subsiste la representación de la regularidad.

En la época de Weber, pues, la noción de ciencia natural, restringida al enunciado de regularidades representables matemáticamente, era dominante y fue, con propiedad, un obstáculo epistemológico insuperable para el sociólogo alemán.

Sin embargo, en la primera década del siglo XX estaba gestándose una revolución científica de incalculables consecuencias. La obra de Einstein, como enseña Rolando García, no sólo ponía en cuestión al conocimiento físico en su momento, sino la pretendida validez general del paradigma newtoniano. Son los conceptos fundamentales - tiempo, espacio...- los que son puestos en cuestión.

El resultado de esta crisis produce una consecuencia que Einstein advierte con toda claridad; la relevancia de la teoría, como esquema general de interpretación 
de los fenómenos físicos, ocupa el lugar central, desplazando a la leyenda previa que ubicaba en el centro a la observación y experimentación de las cuales, sin más, se inferían las regularidades o leyes. Y con más radicalidad, es el propio Einstein quien postula que los conceptos básicos de las teorías científicas — de la física para ser más precisos - son "creaciones libres del intelecto humano", pues no provienen de la "simple" observación y no hay modo de reducirlos a ella.

¿Quién imaginaría semejante convergencia con Max Weber?:

No las conexiones "de hecho" entre "cosas" sino las conexiones conceptuales entre problemas están en la base de la labor de las diversas ciencias. Una nueva ciencia surge cuando se abordan nuevos problemas con métodos nuevos y, por esta vía, se descubren verdades que inauguran nuevos problemas significativos ${ }^{8}$

La validez de todo saber empírico descansa:

...en esto, y sólo en esto: que la realidad dada se ordene según categorías que son subjetivas en un sentido especifico, en cuanto representan el presupuesto de nuestro conocimiento y están ligadas al presupuesto del valor de aquella verdad que sólo el saber empírico puede proporcionarnos ${ }^{9}$

Pues la ciencia como actividad valorada socialmente, lo que puede otorgar, son:

...conceptos y juicios que no son la realidad empírica, ni la copian, pero que permiten ordenarla conceptualmente de manera válida ${ }^{10}$

Igual que Einstein en un dominio científico tan distinto, Weber reconoce que:

A fin de penetrar acabadamente los nexos causales reales, construimos nexos irreales ${ }^{11}$

En pocas palabras, la propia física — en su desarrollo histórico en el siglo $\mathrm{XX}$ - no se conforma con el horizonte angosto y de corto plazo del establecimiento de re-

${ }^{8}$ Idem.

${ }^{9}$ Idem.

${ }^{10}$ Idem.

${ }^{11}$ Max Weber, "Estudios críticos sobre la lógica de las ciencias de la cultura" (1906) en Ensayos sobre metodología sociológica, Amorrortu, Buenos Aires, 1993. 
gularidades, sino que aspira a establecer leyes teóricas que permitan no sólo reducir los fenómenos particulares, sin contradicción, a una regularidad bien representada, sino a ubicar esa regularidad en un conjunto sistemático, interpretable, de relaciones teóricas. Einstein advierte el asunto, y concluye que la relación entre las formulaciones matemáticas y los fenómenos es, más bien, milagrosa...

Entonces ha sido el propio desarrollo de las ciencias, y su estudio como laboratorio epistemológico, el que ha refutado la solución weberiana de las dos esferas con diversa sed de explicación causal.

Ahora bien una cosa es mostrar que una solución es insuficiente, y otra - bastante distinta y no automática - es contar con una teoría del desarrollo cognitivo que explique la convergencia de todas la ciencias en el esfuerzo de explicación causal. Es aquí donde al análisis histórico le acompaña una mirada epistemológica específica.

Piaget y García ${ }^{12}$ sintetizan una de las tesis centrales del constructivismo genético: la continuidad funcional de los instrumentos, mecanismos y procesos cognitivos:

- No hay discontinuidad funcional del proceso cognitivo con el proceso de la vida

- No hay discontinuidad funcional en el desarrollo cognitivo del individuo en el eventual pasaje de su desarrollo pre-científico a su dedicación especializada en la actividad científica

- No hay, pues, razones para una discontinuidad funcional entre las diversas ciencias de tal suerte que hubiese algo así como el sujeto epistémico de las naturales y otro para las sociales o las formales.

- No se postula - y esto es crucial para evitar muchos equívocos- continuidad estructural ni en las diversas etapas del desarrollo, ni entre las diversas ciencias: hay, por supuesto, variaciones, pero en razón a la complejidad de las estructuras de asimilación y acomodación en juego — la solidez de sus teorías - en atención a la complejidad de los procesos de abstracción reflexiva y diferenciación, correspondientes a la consolidación variable de sus marcos teóricos generales.

12 Jean Piaget y Rolando García, Psicogénesis e historia de la ciencia, Siglo XXI editores, México, 1992. 
Se postula, pues, se acepta y se reconoce, desde el constructivismo, una gama de variación considerable entre dominios diversos, y al interior mismo de las subdisciplinas, pero no provenientes de insalvables demarcaciones funcionales entre etapas y ciencias específicas.

El sujeto epistémico del constructivismo es un sujeto activo, constructor no sólo de su saber, sino de las estructuras cognitivas que le permiten lograrlo, en todos los niveles de su desarrollo y en las distintas actividades científicas. Es un variante funcional, teóricamente fundado, con toda la apertura necesaria a la diversidad estructural de sus procesos específicos.

Soy consciente de la brevedad de esta descripción de parte del núcleo duro del constructivismo genético, pero contamos con una razón teórica para la convergencia en la sed de explicación causal: uno de los mecanismos fundamentales hallados luego de décadas de trabajo empírico en la sicogénesis y de reflexión sobre la historia de las ciencias, consiste en lo que Piaget y García llaman la incoercible búsqueda de razones, propia del proceso cognitivo y poderoso motor en el proceso de equilibración —y desequilibración, enfatiza García - de las estructuras cognitivas.

Desde el constructivismo genético, igual — supongo — que desde otras tradiciones epistemológicas contemporáneas, pero con una teoría específica del desarrollo cognitivo frecuentemente ausente de los postulados constructivistas en general, hay certeza de la relevancia de la dimensión teórica en toda actividad científica: la simple descripción de las regularidades empíricas no sacian la sed de explicación causal, a menos que se trate no de un científico, sino de un operador automático de destrezas y saberes - un técnico — que se conforma con aplicar, medir y publicar — as soon as posible - las nuevas reacciones; y a pesar de que, por ello, obtenga muchos méritos, puntos y fama en su tribu...

Más allá de la confirmación de la regularidad, permanecen abiertas muchas preguntas que generan la "invención" de "observables", y esto vale en todos los órdenes o escalas del proceso cognitivo: desde el caso de los niños que postulan la existencia de un dueño de la escuela — al que nadie ha "visto" - para cerrar, en cierta etapa del desarrollo de esta noción, su explicación de la autoridad escolar en las escuelas públicas argentinas, cuestión que ha indagado Castorina, hasta el más prestigioso caso de los astrofísicos de nuestros tiempos, quienes postulan la existencia de materia oscura para hacer consistente su aproximación teórica a la masa total del universo. 
En todos los casos, las regularidades son - en otro nivel - interpretadas a través del proceso de atribución a los objetos de la necesidad propia de la lógica de las teorías ${ }^{13}$. La regularidad de la dilatación de los metales por el calor, encuentra, en otro nivel, su interpretación teórica, cuestión que ocurre también con las leyes - regularidades - en el comportamiento de los gases, o bien, en otro orden de fenómenos, la desaparición de la moneda cuyo valor material es mayor al nominal — regularidad observada en diversos momentos de la historia económica - resulta comprensible desde la teoría económica del actor como maximizador de sus ganancias mediante el cálculo.

\section{VII}

\section{A manera de conclusión y apertura al diálogo}

Por todo lo anteriormente expuesto, sostengo que no es necesaria, ni conveniente, una propuesta dicotómica entre los procesos epistemológicos básicos en la construcción de los objetos de conocimiento de las ciencias naturales y las históricosociales y tampoco en las actividades científicas no empíricas, tales como la matemática y la lógica. Ni las primeras se "inician" — como se repite tanto en las escuelas - con la observación y la experimentación, ni las segundas se constituyen mediante "vivencias" y las terceras, pese a lo que los matemáticos suelen afirmar, no proceden del topos uranos. En todas las actividades científicas, la descentración es necesaria y esto implica el espacio para la constancia de estructuras teóricas en el origen de todas las aproximaciones a los fenómenos que nos interesa explicar y comprender.

Y son las preguntas, como mostró Piaget a lo largo de sus obras, el interés cognitivo al que aludía Weber, la piedra de toque para entender la variedad, la diversidad de sus desarrollos históricos. En todos los casos, se cumple el dictum constructivista, apoyado por miles de evidencias empíricas: "uno no sabe lo que ve; uno ve lo que sabe", ya intuido por Goethe y citado por Weber: “...para decirlo con Goethe, el "hecho" incluye ya "teoría". ${ }^{14}$

\footnotetext{
${ }^{13}$ Así sea de manera muy sintética, éste es el proceso explicativo de la posibilidad de enunciados causales proveniente de las indagaciones piagetianas. La fuerza y necesidad de las relaciones lógicas, de las deducciones propias de los sistemas teóricos, son atribuidas por el sujeto investigador a las relaciones entre los objetos.

${ }^{14}$ Weber hace esta cita a Goethe en el ensayo de 1906, ver supra, p. 160.
} 
La construcción de los objetos de conocimiento, en las diversas ciencias, dado que el sujeto que investiga, en sus condiciones epistemológicas fundamentales, no es variable según el objeto que atiende, implican una actividad fundamental del sujeto, igualmente refutadora del empirismo y de los varios tipos de apriorismo. En todos los casos, como muestra el constructivismo genético en sus indagaciones, el "objeto" se resiste, recula, puesto que la realidad, en efecto, es organizable a través de nuestras teorías, pero no de cualquier manera.

Finalmente, creo que es posible revisar la opinión actual que divide a las ciencias entre ciencias y "ciencias sociales", o la semejante que prefiere adjetivar en duras y blandas a las distintas ramas de la actividad científica. Hay, tal vez, concepciones epistemológicas bien fundadas y otras que, en lugar de enfrentar epistemológicamente los problemas, los eluden asociándose a dicotomías hace un siglo - al menos - superadas.

Reorganizar, desde el constructivismo, no implica resolver los problemas, pues éstos siempre están abiertos; significa un intento de plantearlos bien, de tal modo que puedan ser sometidos al escrutinio de su coherencia lógica y su relación fértil con los fenómenos a los que aluden. Con esta convicción de base, he redactado estas reflexiones y con una adicional: prefiero la comunicación entre los científicos, y los estudiosos de los fundamentos de la ciencia, sin arrancar con dicotomías o demarcaciones insalvables.

P.D. Otra manera de observar el saldo metodológico de Weber es la siguiente:

\section{El saldo metodológico de Weber}

Luego de revisar con detalle los trabajos metodológicos de Weber ${ }^{15}$, disponibles en español, podemos sintetizar sus conclusiones del siguiente modo:

a. Las ciencias histórico-sociales han de concebirse, con toda propiedad, como ciencias. Rechaza, entonces, dos tendencias muy vivas en su tiempo y tradición intelectual: la dicotomía radical entre ciencias de la naturaleza y ciencias del espíritu y, sobre todo, la supuesta incalculabilidad esencial de la acción social en comparación con la también supuesta calculabilidad absoluta de los hechos naturales.

${ }^{15}$ El reporte pormenorizado de esta labor se encuentra en: Manuel Gil Antón, Conocimiento científico y acción social, crítica epistemológica a la concepción de ciencia en Max Weber, Gedisa, Barcelona, 1997 
b. Las ciencias histórico-sociales, para cumplir a cabalidad con su condición de ciencias, no deben reducir sus procedimientos al protocolo establecido - en ese entonces - por las ciencias de la naturaleza, expresable de manera harto resumida como la elaboración conceptual que descansa en el saber previo que guía a la observación y de ahí procede, por inferencias y generalizaciones, a la posibilidad de constatación de regularidades.

c. Las ciencias no se distinguen entre sí por la "tasa" de calculabilidad de los fenómenos que estudian, ni por cualquier otra atribución a la "naturaleza" de sus objetos, sino por elinterés cognitivo específico que anima a los sujetos que las realizan. Puesta la fuente de la distinción entre las ciencias del lado del sujeto, el interés cognitivo específico implicará diversas formas de elaboración conceptual: a su juicio, en la esfera del conocimiento propia de las ciencias de la naturaleza, la sed de explicación causal se sacia cuando un caso particular se incluye, sin contradicción, en el horizonte establecido de las regularidades observadas. En cambio, en la esfera del conocimiento de las ciencias históricosociales, el interés cognitivo que las anima y su sed causal específica las conduce a que, además del trabajo con base en las regularidades observadas, deba realizarse un esfuerzo de interpretación del sentido de la acción. En esta esfera del conocimiento, correspondiente al interés cognitivo de las ciencias de la acción social, la elaboración conceptual requiere una compleja relación entre el esquema causa-efecto y el esquema medios-fines, para arribar a la explicación comprensiva de los fenómenos.

d. A su entender, en todas las ciencias, pero sobre todo en las histórico-sociales, la elaboración de los conceptos es crucial e implica una muy profunda actividad del sujeto; esto es, los conceptos no son una copia de la realidad, sino el resultado de una selección - y relación - teórica de los elementos cruciales por destacar entre los múltiples que intervienen en los fenómenos. Y la función del concepto elaborado en la esfera de los fenómenos interpretables - el concepto típico ideal- no consiste en ser patrón de ajuste de los hechos a explicar, sino en mantener su distancia del acaecer efectivo de manera constante - debido al caracter constructivo (ficticio) y la lógica de la estricta racionalidad en su elaboración - con el fin de que, a contra luz de su coherencia, el investigador pueda realizar hipótesis específica de los motivos y sentidos - racionales o no racionales - involucrados efectivamente en el proceso que intenta comprender explicativamente. 
e. Si los conceptos no son la realidad, ni su copia, sino estructuras de ordenación de la realidad elaboradas por el sujeto, permiten al investigador, al contrastarlo con el fenómeno por explicar, evaluar la relevancia causal de diversos factores: ¿habría ocurrido lo mismo sin la presencia de determinado elemento? Si se afirma, su relevancia causal tiende a cero; si se niega, se puede establecer su relevancia causal en la generación del fenómeno a explicar comprensivamente.

No es posible, ni de lejos, aspirar a una síntesis completa del esfuerzo metodológico weberiano en un par de cuartillas. Estos cinco rasgos, sin embargo, forman parte del saldo conseguido y permiten describir la concepción de ciencia histórico-social que postula posible y a la que procurará ajustarse en sus indagaciones: ciencia en sentido estricto, más allá — sin despreciar su valor— del análisis de regularidades observadas al incluir la vía de la interpretación de tal suerte de conseguir la comprensión explicativa de los fenómenos. Es un proceder científico plenamente consciente de la elaboración de los conceptos por parte del investigador, procurando la máxima coherencia lógica en su construcción — evidencia-y dejando al análisis del contraste con la información el proceso de la validez de las hipótesis que, a contra luz, sea factible establecer. De este modo, la propuesta weberiana se abre al espacio de verificación empírica de la relevancia causal de los elementos elegidos como importantes.

Como muy bien expone Hughes ${ }^{16}$, Weber pertenece a la generación de científicos sociales que sostuvieron propuestas de actividad científica en un contexto en el cual ya formaba parte del saber sobre los hombres la formidable teoría de Freud sobre el inconsciente. Rota la aspiración a la racionalidad absoluta derivada de la Ilustración, acotada si se quiere, o abolida en la visión del mundo social predominante, resulta mucho más valiosa y sorprendente la batalla weberiana para sostener un sistema de teorización racional con el cual enfrentar, explicar y comprender los hechos sociales incluyendo la no racionalidad como parte de sus características. Más allá de sus logros efectivos, la empresa no era menor ni triviales sus consecuencias $^{17} \cdot \mathrm{d}$

\footnotetext{
${ }^{16}$ H. Stuart Hughes: Conciencia y sociedad: la reorientación del pensamiento social europeo 1890-1930, Aguilar, Madrid, 1972. La edición inglesa original corresponde a 1958. Agradezco mucho a Ricardo Yocelevzky su insistente sugerencia de atender a esta obra: como siempre, he quedado en deuda con su buen juicio para leer obras relevantes.

${ }^{17}$ La guía con la cual emprendí en su momento el estudio de los ensayos metodológicos de Weber, y a la cual con frecuencia retorno, es la obra de Luis F. Aguilar Villanueva, Weber: La idea de ciencia social, volumen primero: La tradición, volumen segundo: La innovación, Coordinación de Humanidades, UNAM, y Miguel Ángel Porrúa, Grupo Editorial, México, 1988.
} 